\title{
Confirming the appearance of excess success: Reply to van Boxtel and Koch (2016)
}

\author{
Gregory Francis ${ }^{1}$
}

Published online: 25 August 2016

(C) Psychonomic Society, Inc. 2016

\begin{abstract}
Boxtel and Koch (Psychonomic Bulletin \& Review. doi:10.3758/s13423-016-1010-0, 2016) reported finding problems in the Test for Excess Success (TES) analysis in Francis (Psychonomic Bulletin \& Review, 21, 1180-1187, 2014). They argued that their findings undermined the general analysis and the conclusions of the specific TES analysis for their article (van Boxtel \& Koch in Psychological Science, 23(4), 410-418, 2012). As shown in this paper, their reported problems reflect misunderstandings about both the general properties of a TES analysis and how it was applied to their specific set of findings. Another look at the findings and theoretical claims in van Boxtel and Koch (Psychological Science, 23(4), 410-418, 2012) confirms the appearance of excess success.
\end{abstract}

Keywords Statistics $\cdot$ Statistical inference $\cdot$ Visual perception

Van Boxtel and Koch (2016) reported finding problems in the Test for Excess Success (TES) analysis in Francis (2014). They argued that their findings undermined the general analysis and the conclusions of the specific TES analysis for their article (van Boxtel \& Koch, 2012). As shown below, their reported problems reflect misunderstandings about both the general properties of a TES analysis and how it was applied to their specific set of findings. Another look at the findings and theoretical claims in van Boxtel and Koch (2012) confirms the appearance of excess success.

Gregory Francis

gfrancis@purdue.edu

1 Department of Psychological Sciences, Purdue University, West Lafayette, IN, USA
A TES analysis uses reported data to estimate the probability that a set of replication studies with the same sample sizes as the original experiments would produce results at least as successful as the original set. In situations where scientists consider replication to be important, one ideally wants a high estimated probability of replication success. (There are situations where replication is not important, such as when a single counterexample can disprove a theory.) The TES probability calculation supposes that reported results are free of publication bias and questionable research practices (QRPs) that potentially undermine the validity of conclusions from experimental data (John, Loewenstein \& Prelec, 2012; Kerr, 1998; Simmons, Nelson, \& Simonsohn, 2011). Similar to hypothesis testing, when the success probability is low, readers should be skeptical that the original experiment set was free of publication bias and QRPs (Ioannidis \& Trikalinos, 2007; Schimmack, 2012), and thus readers should doubt the conclusions that were derived from the data.

\section{Point 1: The TES analysis does not require all experiments to be replications}

Although Ioannidis and Trikalinos (2007) developed the TES by using meta-analytic techniques across replication experiments to estimate a pooled effect size that was then used to calculate success probabilities, the logic of the TES does not require this approach (Francis, 2013). Using a pooled effect size makes sense when the theoretical claim is simply a statement about the existence and magnitude of an effect. In other cases, as for many of the analyses reported in Francis (2014), a theoretical claim is based on multiple outcomes that act as control conditions to rule out alternative theories, establish boundary 
conditions for an effect, or provide converging evidence from several different types of investigations. Sometimes these different outcomes involve multiple tests within a single experiment, and other times they involve a pattern of results across different experiments.

Van Boxtel and Koch (2016) refer to Ioannidis (2013) when claiming that the TES analysis is valid only when all the tests investigate the same hypothesis, but the claim is not true, and Ioannidis (2013) makes no such statement. Van Boxtel and Koch (2016) also claimed that the analyses in Francis (2014) used "a single overall average effect size to estimate power." Contrary to their claim, the supplemental material of Francis (2014) explains how the success probabilities were computed with simulated experiments that used the sample statistics as estimated population parameters. The supplemental material of Francis (2014) includes source code for these simulations, so readers can examine the details of the analysis for themselves.

\section{Point 2: Selecting which tests to include in the TES analysis is straightforward}

The primary concern of van Boxtel and Koch (2016) is that Francis (2014) mistakenly identified the tests that should be included in a TES analysis of their findings. They feel that the TES analysis in Francis (2014) included tests that were tangential to their theoretical claims. Fortunately, this disagreement can be resolved because the relevant tests were clearly identified in van Boxtel and Koch (2012). The relation between the theoretical claims and the reported experiments and tests are summarized in Table 1, which simply restates the reasoning provided in van Boxtel and Koch (2012). For example, Experiment 1 made the theoretical claim that object-based processing, rather than retinal spatial processing, determines rivalry occurrence. Van Boxtel and Koch (2012) presented a total of eight experimental outcomes to support their theoretical claim, and the need for these tests relative to their theoretical claim was clear in their main text. Namely, conditions $\mathrm{O}+\mathrm{R}+$ and $\mathrm{O}-$

Table 1 Relevant theoretical claims, the supporting tests, and the reasons the test are needed for the findings in van Boxtel and Koch (2012). NS stands for nonsignificant

\begin{tabular}{|c|c|c|c|}
\hline Theoretical claim & Supporting tests & Analysis & Reason test is needed \\
\hline \multirow[t]{3}{*}{$\begin{array}{l}\text { Exp. 1: Object-based processing, rather than } \\
\text { retinal spatial processing, determines rivalry } \\
\text { occurrence. }\end{array}$} & $\begin{array}{l}\mathrm{O}+\mathrm{R}+\text { vs. } 1 \\
\mathrm{O}+\mathrm{R}+\text { vs. } 0\end{array}$ & $t$ tests & $\begin{array}{l}\text { Control that produces normal rivalry with no perceptual bias. An NS } \\
\text { effect would suggest a problem with the stimulus } \\
\text { display or task. }\end{array}$ \\
\hline & $\begin{array}{l}\text { O-R- vs. } 0.5 \\
\text { O-R- vs. } 0\end{array}$ & $\begin{array}{l}t \text { test } \\
\text { NS } t \text { test }\end{array}$ & $\begin{array}{l}\text { Control that produces perceptual bias (the NS result) without } \\
\text { rivalry. Other outcomes would suggest a problem with the } \\
\text { stimulus display or task. }\end{array}$ \\
\hline & $\begin{array}{l}\text { O+R- vs. } 0.5 \\
\text { O+R- vs. } 0 \\
\text { O+R- vs. } 1 \\
\text { O-R+ vs. } 0.5\end{array}$ & $\begin{array}{l}\text { NS } t \text { test } \\
t \text { test } \\
t \text { test } \\
t \text { test }\end{array}$ & Indicates that object-based bias overrules retinal influences. \\
\hline \multirow{3}{*}{$\begin{array}{l}\text { Exp. 2: Object-based rivalry is similar to con- } \\
\text { ventional rivalry with regard to spatial } \\
\text { layout. }\end{array}$} & $\begin{array}{l}\text { Main effect of } \mathrm{O}+ \\
\mathrm{R}-\text { and } \mathrm{O}+\mathrm{R}+\end{array}$ & $F$ test & Replicates findings from Exp. 1. \\
\hline & $\begin{array}{l}\text { Main effect of dot } \\
\text { distance }\end{array}$ & $F$ test & Shows effect of spatial layout. \\
\hline & Interaction & $\begin{array}{l}\text { NS } F \\
\text { test }\end{array}$ & Suggests no difference between the stimulus conditions. \\
\hline \multirow[t]{3}{*}{$\begin{array}{l}\text { Exp. 3: Object-based rivalry depends on the } \\
\text { quartet being perceived as stationary in } \\
\text { the object reference frame. }\end{array}$} & $\begin{array}{l}\text { Group motion vs. } \\
\quad 0.5 \\
\text { Group motion vs. } 0\end{array}$ & $\begin{array}{l}\text { NS } t \text { test } \\
t \text { test }\end{array}$ & $\begin{array}{l}\text { Stationary quartet in the object reference frame demonstrates } \\
\text { rivalry without bias. }\end{array}$ \\
\hline & $\begin{array}{l}\text { Element motion vs. } \\
\quad 0.5\end{array}$ & $t$ test & $\begin{array}{l}\text { Motion of the quartet in the object reference frame demonstrates } \\
\text { bias without rivalry. }\end{array}$ \\
\hline & $\begin{array}{l}\text { Group motion vs. } \\
\text { element motion }\end{array}$ & $t$ test & Demonstrates differences between the conditions. \\
\hline \multirow[t]{3}{*}{$\begin{array}{l}\text { Exp. 4: Rivalry is object based rather than } \\
\text { object centered. }\end{array}$} & $\begin{array}{l}\text { Group motion vs. } \\
0.5 \\
\text { Group motion vs. } 0\end{array}$ & $\begin{array}{l}t \text { test } \\
\text { NS } t \text { test }\end{array}$ & $\begin{array}{l}\text { Control condition to rule out object-centered explanation for Exps. } \\
1-3 \text {. }\end{array}$ \\
\hline & $\begin{array}{l}\text { Element motion vs. } \\
0.5 \\
\text { Element motion vs. } \\
\quad 0\end{array}$ & $\begin{array}{l}t \text { test } \\
\mathrm{NS} t \text { test }\end{array}$ & $\begin{array}{l}\text { Control condition to rule out object-centered explanation for Exps. } \\
1-3 \text {. }\end{array}$ \\
\hline & $\begin{array}{l}\text { Group motion vs. } \\
\text { element motion }\end{array}$ & NS $t$ test & No evidence of difference between these conditions. \\
\hline
\end{tabular}

Note. $\mathrm{O}$ and $\mathrm{R}$ indicates the presence (+) or absence (-) of object-based or retinal-based reference frames 
R- were control conditions that produced rivalry without bias or bias without rivalry. Had these conditions produced some other pattern of results, it would have indicated a flaw in the stimulus, task, or instructions to the observers, and would thereby have undermined the theoretical claim. Conditions $\mathrm{O}+\mathrm{R}$ - and $\mathrm{O}-\mathrm{R}+$ demonstrated that rivalry could occur without retinal conflict and that object-based processing could dominate retinal-based processing effects. Again, failure for either of the results would have undermined the theoretical claims made in van Boxtel and Koch (2012).

Van Boxtel and Koch (2016) presents somewhat conflicting views of their original experimental design. On the one hand, they properly explain how they expected, or not, to find rivalry or bias in different situations; but on the other hand they claim that only one test $(\mathrm{O}+\mathrm{R}-)$ was actually of interest to them and that other tests were "immaterial to the hypothesis under scrutiny." Such a statement undermines their original reasoning; and if it were an accurate representation of their theoretical conclusions, then readers must wonder why they gathered, reported, and described so many irrelevant data outcomes. Table 1 provides a similar summary of the reasoning described in van Boxtel and Koch (2012) for how their reported tests for Experiments 2-4 related to their theoretical claims.

Van Boxtel and Koch (2016) suggested that Experiment 4 was a special case because it was exploratory and therefore should not be part of the TES analysis; but whether the experiment was exploratory or confirmatory hardly matters because either way its results formed the basis for a theoretical claim about rivalry being object based rather than object centered. From the perspective of the TES analysis, the problem is not that van Boxtel and Koch (2012) derived their theory after observing the data, but that the data provided only weak support for the theoretical claim (the success probability estimate is 0.364 ).

In summary, the selection of tests to include in the TES analysis of van Boxtel and Koch (2012) followed the welldescribed reasoning of the authors. A failure of any of the tests would have undermined at least some of the theoretical claims in van Boxtel and Koch (2012), and the TES analysis indicates that at least one failure should be rather likely for studies like these. With the current data, there is no way to identify which theoretical claims seem valid and which seem invalid.

\section{Point 3: $P$ curve is a poor choice for analyzing the findings in van Boxtel and Koch (2012)}

Van Boxtel and Koch (2016) performed a $p$-curve analysis of their findings (Simonsohn, Nelson, \& Simmons, 2014) as a way of providing some support that they did not " $p$-hack." However, the findings in van Boxtel and Koch (2012) seem inappropriate for a $p$-curve analysis because this kind of analysis can only use findings from independent tests. To satisfy this requirement, only a single test from Experiment 1 could be part of the $p$-curve analysis, even though the theoretical claim depended on multiple successful tests. The same problems apply to the $p$-curve analyses for other papers reported in Table S1 of van Boxtel and Koch (2016).

The TES analysis does not deny that some of the findings in van Boxtel and Koch (2012) appear to have some evidential value. However, an experiment set can simultaneously have some evidential value and excess success. In a similar way, it is possible for a full set of theoretical claims to be invalid even if some (unknown) subset of the claims is true.

\section{Point 4: The calculations for power used by van Boxtel and Koch (2016) are not correct}

The TES analysis requires an estimate of success probabilities for the tests that are relevant to the theoretical claims. Van Boxtel and Koch (2016) introduced several estimation methods for the tests in Experiment 1 of van Boxtel and Koch (2012). Although quantitatively similar to the values reported in Francis (2012), their estimation methods are invalid. In the present context, these errors are a minor point, but van Boxtel and Koch (2016) suggest that their calculations are "more direct" than those in Francis (2014), and I want to warn scientists against repeating their mistakes in other situations where the errors may not be minor.

When estimating the probability of success for all of the tests in Experiment 1, van Boxtel and Koch (2016) multiplied the success probabilities of the individual tests. This procedure would be appropriate if the tests were independent, but some tests involved the very same measurement being compared against different values (e.g., the measure for $\mathrm{O}+\mathrm{R}$ - is compared against $0,0.5$, and 1 , to test for horizontal bias, rivalry, and vertical bias, respectively). Because a random sample of data can only produce one measurement (sample mean), the outcomes of these tests are related to each other (the tests are not independent), so multiplying their individual success probabilities to produce a probability for joint success is not correct. Francis (2014) ran simulated experiments and thereby directly estimated the joint success probability — an approach that takes into account the dependence of the multiple tests.

Van Boxtel and Koch (2016) subsequently calculated another estimate of success probability by averaging the success probabilities of the different tests and then using the properties of the binomial distribution to compute the probability of observing success in all eight tests. This averaging process is inappropriate, as can be seen by an example. Suppose I take one card from each of two standard shuffled decks. I am interested in the joint probability that the draw from the first deck is the queen of hearts and that the draw from the second deck is not a king. The individual probabilities are easily calculated, $P($ queen of hearts from deck 1$)=1 / 52=0.019 ; P($ not 
king from deck 2) $=48 / 52=0.923$. The average probability of these two events is 0.471 , and the binomial probability for two events with the average probability is $(0.471)^{2}=0.222$. In contrast, the proper calculation of the joint probability for the two events is the product of the individual probabilities, which is 0.018 . As it turns out, for the success probabilities used in van Boxtel and Koch (2012), the binomial calculation produces a joint success probability $(0.56)$ that is nearly the same as the proper calculation (0.52) presented in Francis (2014), but other scientists should not repeat their error when calculating joint probabilities.

Van Boxtel and Koch (2016) next suggested that the estimated joint success probability "poorly reflects the strength of the findings," apparently because their calculated $p=.56$ does not indicate a significant effect and is much smaller than the average power (0.93). In addition to mistakenly interpreting the estimated joint success probability as a $p$ value, van Boxtel and Koch (2016) seem to have fallen victim to the "conjunction fallacy," which violates the fundamental property of probability that a joint set of outcomes must always be no more probable than any subset of those outcomes (e.g., Tversky \& Kahneman, 1983). The estimated joint success probability does accurately reflect the strength of the experimental findings, and there are so many findings needed to support the full set of theoretical claims that their overall strength is rather poor.

\section{Conclusion}

Setting aside the calculation errors of success probabilities in van Boxtel and Koch (2016), their primary disagreement with the analysis in Francis (2014) concerns which tests were relevant to the theoretical claims in van Boxtel and Koch (2012). Readers will have to check the text of van Boxtel and Koch (2012) to verify that the description of the tests in Table 1 properly represents their original reasoning.

Despite the appearance of excess success in van Boxtel and Koch (2012), there are reasons to believe that better-designed experiments or better analyses of the original design could find evidence of object-based processing of visual motion rivalry. Earlier work found that apparent motion percepts involved nonretinotopic (object-based) processing rather than retinal-based processing (Boi, Öğmen, Krummenacher, Otto, \& Herzog, 2009; see also a demo at http://lpsy.epfl.ch, recent modeling by Clarke, Öğmen, \& Herzog, 2015, and a recent fMRI study by Thunell, van der Zwaag, Öğmen, Plomp, \& Herzog, 2016), so it would be somewhat surprising if ambiguous apparent motion rivalry did not have similar characteristics.
Although there is optimism for future work on this topic, the original conclusion from Francis (2014) remains: the findings in van Boxtel and Koch (2012) are rather unbelievable if the true effects were as reported, the experiments and analyses were done properly, and all relevant studies were reported. Thus, readers should remain skeptical of the findings and theoretical claims in van Boxtel and Koch (2012).

Acknowledgments The author thanks Michael Herzog for helpful feedback on a draft of this article.

\section{References}

Boi, M., Öğmen, H., Krummenacher, J., Otto, T. U., \& Herzog, M. H. (2009). A (fascinating) litmus test for human retino- vs. non-retinotopic processing. Journal of Vision, 9(5), 1-11. doi:10.1167/9.13.5

Clarke, A. M., Öğmen, H., \& Herzog, M. H. (2015). A computational model for reference-frame synthesis with applications to motion perception. Vision Research. doi:10.1016/j.visres.2015.08.018

Francis, G. (2013). Replication, statistical consistency, and publication bias. Journal of Mathematical Psychology, 57, 153-169.

Francis, G. (2014). The frequency of excess success for articles in Psychological Science. Psychonomic Bulletin \& Review, 21, 1180-1187.

Ioannidis, J. P. A. (2013). Clarifications on the application and interpretation of the test for excess significance and its extensions. Journal of Mathematical Psychology, 57(5), 184-187.

Ioannidis, J. P. A., \& Trikalinos, T. A. (2007). An exploratory test for an excess of significant findings. Clinical Trials, 4, 245-253.

John, L. K., Loewenstein, G., \& Prelec, D. (2012). Measuring the prevalence of questionable research practices with incentives for truthtelling. Psychological Science, 23, 524-532.

Kerr, N. L. (1998). HARKing: Hypothesizing after the results are known. Personality and Social Psychology Review, 2, 196-217.

Schimmack, U. (2012). The ironic effect of significant results on the credibility of multiple study articles. Psychological Methods, 17(4), 551-566.

Simmons, J. P., Nelson, L. D., \& Simonsohn, U. (2011). False-positive psychology: Undisclosed flexibility in data collection and analysis allows presenting anything as significant. Psychological Science, 22, 1359-1366.

Simonsohn, U., Nelson, L. D., \& Simmons, J. P. (2014). P-curve: A key to the file drawer. Journal of Experimental Psychology: General, 143(2), 534-547.

Thunell, E., Van Der Zwaag, W., Öğmen, H., Plomp, G., \& Herzog, M. H. (2016). Retinotopic encoding of the Ternus-Pikler display reflected in the early visual areas. Journal of Vision, 16(26), 1-11. doi:10.1167/16.3.26

Tversky, A., \& Kahneman, D. (1983). Extensional versus intuitive reasoning: The conjunction fallacy in probability judgment. Psychological Review, 90, 293-315.

van Boxtel, J. J. A., \& Koch, C. (2012). Visual rivalry without spatial conflict. Psychological Science, 23(4), 410-418.

van Boxtel, J. J. A., \& Koch, C. (2016). Reevaluating excess success in psychological science. Psychonomic Bulletin \& Review. doi:10.3758/s13423-016-1010-0 